\title{
OPEN Contractility of temporal inverted internal limiting membrane flap after vitrectomy for macular hole
}

\begin{abstract}
Akira Hirata $^{1,2 \bowtie}$, Keiko Mine ${ }^{1} \&$ Ken Hayashi ${ }^{1}$
We investigated the postoperative visual outcomes and morphological changes of the internal limiting membrane (ILM) flap, in patients who underwent the temporal inverted ILM flap technique for macular hole (MH). Between August 2018 and February 2020, 22 eyes of 22 patients with idiopathic or myopic $\mathrm{MH}$ who underwent vitrectomy with ILM flap were included in this study and followed-up for more than 6 months. Postoperative MH status, comparison of best-corrected visual acuity (BCVA) before and 6 months after surgery, changes in the ILM flap area at 1 and 6 months postoperatively, and the factors related to changes in ILM flap size, were analyzed. MH closure was achieved in all of the patients. The BCVA at 6 months postoperatively $(0.18 \pm 0.15)$ was significantly better than the preoperative BCVA of $0.63 \pm 0.37(P<0.001$, paired $t$ test). The area of the ILM flap decreased significantly from $3.25 \pm 1.27 \mathrm{~mm}^{2}$ at 1 month to $3.13 \pm 1.23 \mathrm{~mm}^{2}$ at 6 months $(P=0.024$, Wilcoxon signed-rank test). Two eyes showed an ILM flap contraction of more than $20 \%$, and one eye required reoperation due to an increase in metamorphopsia and decreased visual acuity. Among age, sex, ILM flap area at 1 month, preoperative BCVA, and axial length, ILM flap contraction was correlated with patient age and ILM flap area. Although vitrectomy with the inverted ILM flap technique confers a good visual outcome, the ILM flap may contract in younger patients.
\end{abstract}

Since the usefulness of vitrectomy for macular holes (MHs) was reported by Kelly and Wendel, surgical treatment for $\mathrm{MH}$ has become possible ${ }^{1}$. Furthermore, with the introduction of internal limiting membrane (ILM) peeling, both postoperative anatomical $\mathrm{MH}$ closure and improvement of visual function have been achieved with good and stable results ${ }^{2}$. However, in some cases, such as large $\mathrm{MH}$, long-term $\mathrm{MH}$, and myopic $\mathrm{MH}$, treatment was difficult ${ }^{3}$. Michalewska et al. reported that the ILM flap technique can close refractory $\mathrm{MH}$ and continuously improve the foveal architecture ${ }^{4}$. Subsequent papers also reported that the ILM flap technique is superior in closing refractory $\mathrm{MH}^{5-9}$. The ILM flap technique has been modified from the original technique by Michalewska et al., which might more appropriately be called "ILM insertion," to various other techniques, including the creation of a single ILM flap covering the $\mathrm{MH}^{10,11}$.

On the other hand, placing the ILM flap over the macula may create a scaffold for cell proliferation, which may lead not only to $\mathrm{MH}$ closure but also to epiretinal membrane-like proliferative tissue formation. In our previous report, we described a case of $\mathrm{MH}$ with macular pucker due to contraction of a single sheet of the ILM flap created from the temporal side after vitrectomy with silicone oil injection ${ }^{12}$. However, there are no other reports on the changes that occur in the ILM flap after vitrectomy with gas tamponade.

With the advancement of optical coherence tomography (OCT), it has become possible to capture changes in the fine structure of the retina, and to quantitatively observe changes in the vitreous-retinal interface, including the shape of the ILM flap. In this study, we investigated changes in postoperative MH status and visual acuity after vitrectomy for $\mathrm{MH}$, using the inverted ILM flap technique. Furthermore, changes in the ILM flap were compared between 1 month and 6 months after vitrectomy, and the clinical factors affecting the changes in the ILM flap were examined.

\section{Results}

This study included 22 eyes of 22 patients who underwent vitreous surgery for $\mathrm{MH}$. The MHs were idiopathic in 13 eyes and myopic in nine eyes. The clinical characteristics of the patients are summarized in Table 1. Compared to the myopic MH group, the idiopathic MH group was significantly older, had a larger $\mathrm{MH}$ size, and had poor preoperative BCVA.

${ }^{1}$ Hayashi Eye Hospital, 4-23-35, Hakataekimae, Fukuoka, Fukuoka 812-0011, Japan. ${ }^{2}$ Department of Anatomy, Kurume University School of Medicine, 67 Asahi-machi, Kurume, Fukuoka 830-0011, Japan. ${ }^{\varpi}$ email: akhirata@ gmail.com 


\begin{tabular}{|l|l|l|l|c|}
\hline & Total $(\mathbf{n}=\mathbf{2 2})$ & Idiopathic MH $(\mathbf{n}=13)$ & Myopic MH $(\mathbf{n}=9)$ & P-value \\
\hline Patient age (years) & $62.9 \pm 11.0$ & $68.3 \pm 5.9$ & $55.0 \pm 12.1$ & 0.003 \\
\hline Sex (male:female) & $14: 8$ & $9: 4$ & $5: 4$ & 0.660 \\
\hline MH size $(\mu \mathrm{m})$ & $411.1 \pm 178.3$ & $492.5 \pm 172.1$ & $293.4 \pm 112.9$ & 0.007 \\
\hline Preoperative BCVA & $0.63 \pm 0.37$ & $0.81 \pm 0.37$ & $0.37 \pm 0.11$ & 0.001 \\
\hline Axial length $(\mathrm{mm})$ & $25.45 \pm 2.18$ & $24.03 \pm 1.15$ & $27.55 \pm 1.31$ & $<0.001$ \\
\hline
\end{tabular}

Table 1. Patient preoperative clinical characteristics. The values are presented as the mean \pm standard deviation. When comparing the clinical characteristics of patients with idiopathic $\mathrm{MH}$ and myopic $\mathrm{MH}$, an unpaired $t$ test was used for patient age, MH size, preoperative BCVA, and axial length, and Fisher's exact test was used for sex. $M H$ macular hole, BCVA best-corrected visual acuity.
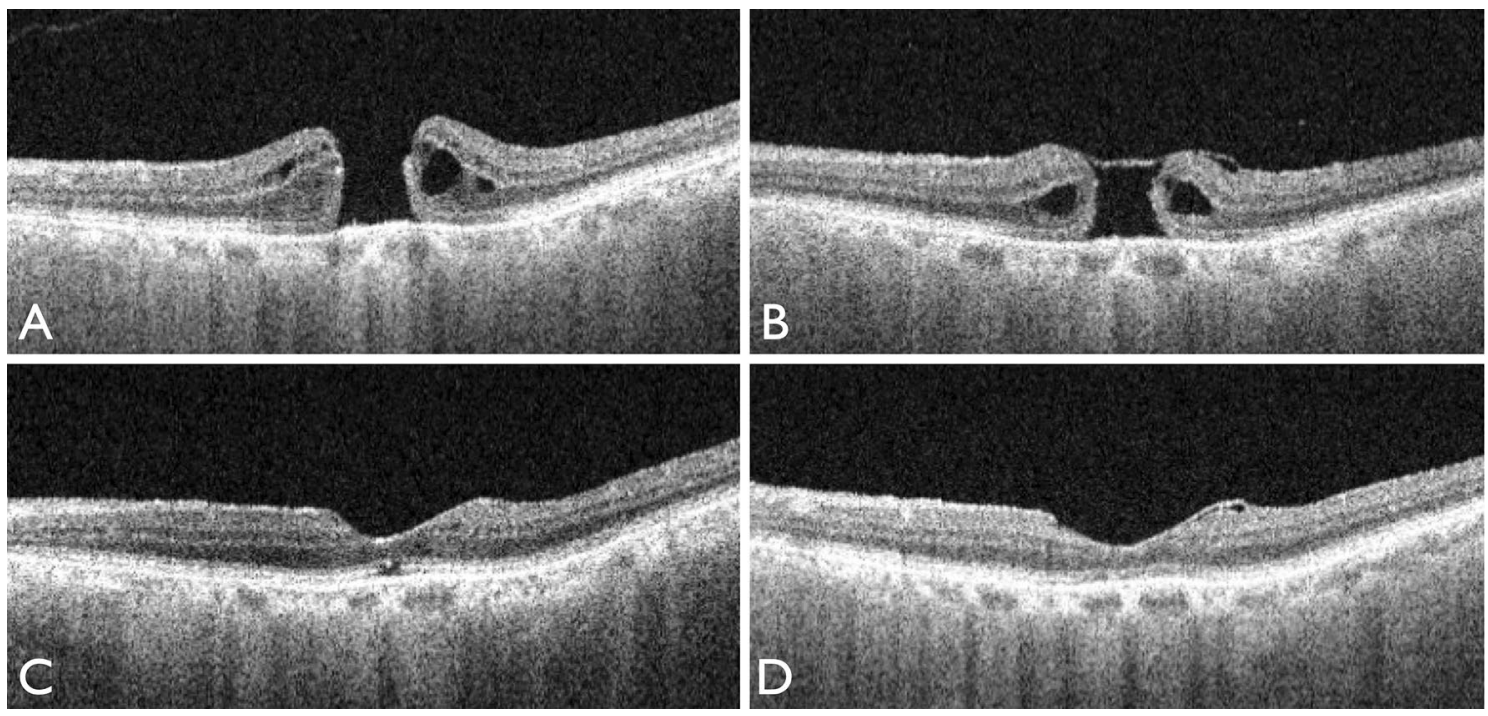

Figure 1. OCT images of a patient before and after macular hole (MH) surgery. (A) Preoperative OCT image. (B) OCT image obtained 1 week after vitrectomy. The hole remained open after the air in the vitreous cavity had disappeared. However, the ILM flap covered the MH. (C) OCT image 1 month after surgery, the MH was closed. However, the ellipsoid zone was disrupted. (D) OCT image at 6 months postoperatively; the continuity of the ellipsoid zone was restored.

$\mathrm{MH}$ closure was observed in 16 of the 22 eyes (73\%) at 1 week after vitrectomy. In the remaining 6 eyes, although the MH was still open, the ILM flap covered the MH. The MH was closed in 21 of the 22 eyes (95\%) at 1 month and in $100 \%$ of the 22 eyes at 1.5 months. Of the 22 eyes, restoration of continuity of the external limiting membrane (ELM) was seen in 4 eyes (18\%) at 1 week, 15 eyes (68\%) at 1 month, and 20 eyes $(91 \%)$ at 6 months. Of the 22 eyes, recovery of the ellipsoid zone continuity was seen in $0 \%$ of eyes at 1 week, 11 eyes $(50 \%)$ at 1 month, and 16 eyes (73\%) at 6 months postoperatively (Fig. 1).

The mean BCVA (log MAR) at 6 months postoperatively was $0.18 \pm 0.16$, which was significantly better than the preoperative BCVA of $0.63 \pm 0.37$ ( $P<0.001$, paired $t$ test; Fig. 2$)$. Correlation analyses revealed that postoperative BCVA after 6 months was significantly correlated with $\mathrm{MH}$ size $(r=0.603, P=0.003)$, preoperative BCVA $(r=0.727, P<0.001)$, and the size of the ILM flap at 1 month $(r=0.468, P=0.028)$. Multivariate linear regression analyses with stepwise regression revealed that preoperative BCVA was a significant factor associated with postoperative BCVA $(\beta=0.727, P<0.001)$.

The ILM flap was clearly observed in the OCT en face image in the vitreoretinal interface region (Fig. 3 ). The area of the ILM flap decreased significantly from $3.25 \pm 1.27 \mathrm{~mm}^{2}$ at 1 month to $3.13 \pm 1.23 \mathrm{~mm}^{2}$ at 6 months $(P=0.024$, Wilcoxon signed-rank test; Fig. 4). Two eyes with idiopathic $\mathrm{MH}$ and three eyes with myopic $\mathrm{MH}$ (a total of five eyes, $23 \%$ ), showed ILM flap contraction of $5 \%$ or more. Two eyes out of the three eyes with myopic $\mathrm{MH}$, had more than $20 \%$ contraction of the ILM flap. In one eye, BCVA decreased, and metamorphopsia was observed with the contraction of the ILM flap. As a result, ILM flap peeling was required (Fig. 5, Supplemental Video 1 and 2). Multiple regression analysis was performed using two factors related to ILM flap contraction: patient age and ILM flap area at 1 month postoperatively, which were selected by a stepwise method among the preoperative and early postoperative clinical findings including sex, patient age, $\mathrm{MH}$ size, preoperative BCVA, axial length, and ILM flap area at 1 month postoperatively. Patient age and the area of the ILM flap at 1 month postoperatively were found to be significant factors related to the contraction of the ILM flap (Table 2). 


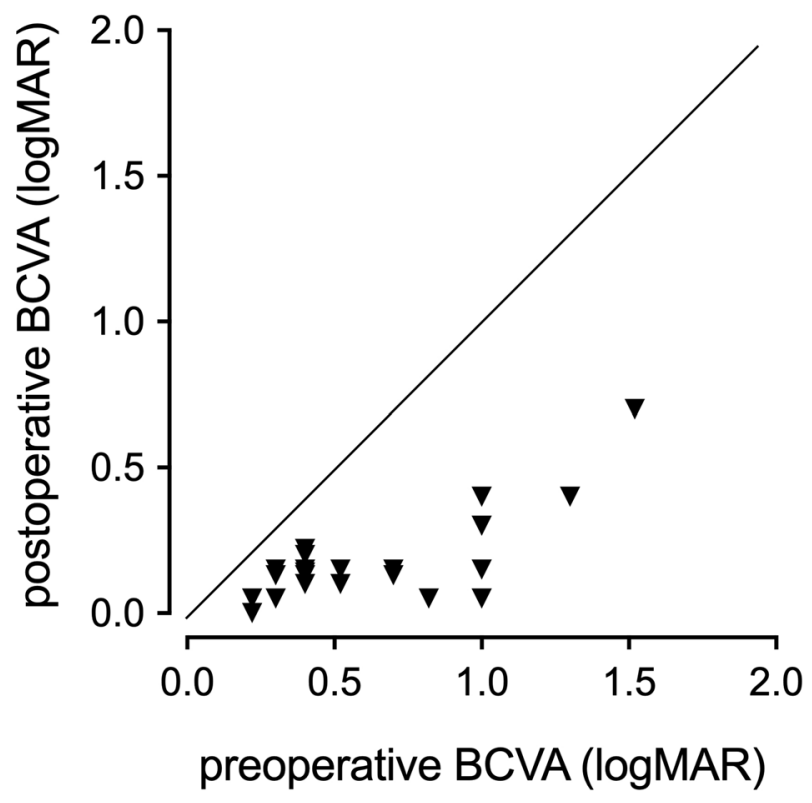

Figure 2. Best-corrected visual acuity (BCVA) before and 6 months after surgery. The preoperative BCVA was $0.63 \pm 0.37$ and the BCVA at 6 months after surgery was $0.18 \pm 0.16$. The postoperative BCVA was significantly improved compared to the preoperative BCVA $(P<0.001$, paired $t$ test).

\section{Discussion}

In this study, we examined the surgical outcomes and postoperative morphological changes of the ILM flap in patients who underwent MH coverage with a hinged ILM flap made on the temporal side after ILM peeling. In all cases, the MH was closed and postoperative BCVA significantly improved. However, in some cases, the ILM flap contracted after the surgery.

The mechanism of MH closure by the ILM flap technique is thought to be multifactorial. The ILM flap may serve as a scaffold for Müller cell proliferation and migration during the $\mathrm{MH}$ closure, and may promote Müller cell activation. Furthermore, neurotrophic factors and bFGF produced by activated Müller cells and present on the surface of the ILM, may contribute to $\mathrm{MH}$ closure $^{13}$. Flap closure has been reported to allow closure of large MHs that would likely remain open without this technique, and to continuously improve the integrity of the foveal structure ${ }^{5}$. In fact, in comparison with ILM peeling, the inverted ILM flap technique showed significantly higher closure rates than a conventional ILM peeling, in MHs greater than $400 \mu \mathrm{m}^{4,14-16}$; in patients with myopic $\mathrm{MH}$, the inverted ILM flap technique has been shown to be more effective than ILM peeling in terms of closure rate, foveal structure, and postoperative $\mathrm{BCVA}^{8}$. In this study, as in previous reports ${ }^{15}$, flap closure was observed in 6 of the 22 eyes (27\%) at 1 week postoperatively, when intravitreal air had disappeared. Subsequently, these 6 eyes gradually achieved $\mathrm{MH}$ closure, without the need for further tamponade, and the outer retinal layers were restored. However, there has been a report of poor anatomical and visual results associated with inverted ILM flaps compared to ILM peeling, suggesting potential limitations of the ILM flap technique for repairing refractory $\mathrm{MH}^{17}$. Between the method of covering the $\mathrm{MH}$ with ILM flap and the inverted ILM flap technique that plugs the $\mathrm{MH}$, the cover group had better anatomical recovery and early postoperative visual acuity ${ }^{18}$. In addition, the method of ILM insertion within the MH had inferior anatomical and visual results compared to the method of ILM flap covering over the $\mathrm{MH}^{19}$. These findings suggest that insertion of the ILM into the MH during MH surgery may interfere with the reconstruction of the outer retinal layer and impede visual recovery ${ }^{20}$.

Michalewska et al. reported the original method of covering the MH with an ILM around the MH, which was followed by the temporal ILM flap which covers the $\mathrm{MH}$ as a single sheet ${ }^{10,11}$, the pedicle ILM transposition flap method ${ }^{21}$, a large semicircular inverted ILM flap ${ }^{22}$, and multiple ILM flaps stacked on top of each other to resemble cabbage leaves ${ }^{23}$. We have devised and used a method of ILM peeling and temporal side ILM flap over the MH. The advantages of this technique are that the ERM around the MH can be completely removed, the ILM flap is large enough to cover the MH, and it can be applied to cases in which ILM peeling was previously performed. As previously reported, it is also possible to create an ILM flap above the $\mathrm{MH}^{24}$.

In contrast, the ILM flap may contract, as shown in this study and in previous reports ${ }^{12,25}$. Multiple regression analysis showed that the younger the age and the larger the ILM flap, the more it tends to contract, and the contraction of the ILM flap may lead to a decrease in postoperative visual function. Studies of epiretinal membrane (ERM) recurrence have shown that ILM peeling reduces ERM recurrence, possibly because of the presence of ERM fragments on the surface of the ILM and the fact that the ILM serves as the basis for cell proliferation and may induce cell proliferation and epiretinal membrane recurrence ${ }^{26-29}$. In this study, histopathological examination revealed that cells adhered to the surface of the ILM that faced the vitreous side after inversion, suggesting that cell proliferation occurred using the ILM flap as a scaffold by a mechanism similar to that of ERM recurrence, resulting in contraction of the ILM flap. As in the case of ERM recurrence, peeling of the ILM flap can be expected to improve visual function in patients with visual impairment ${ }^{12}$. 

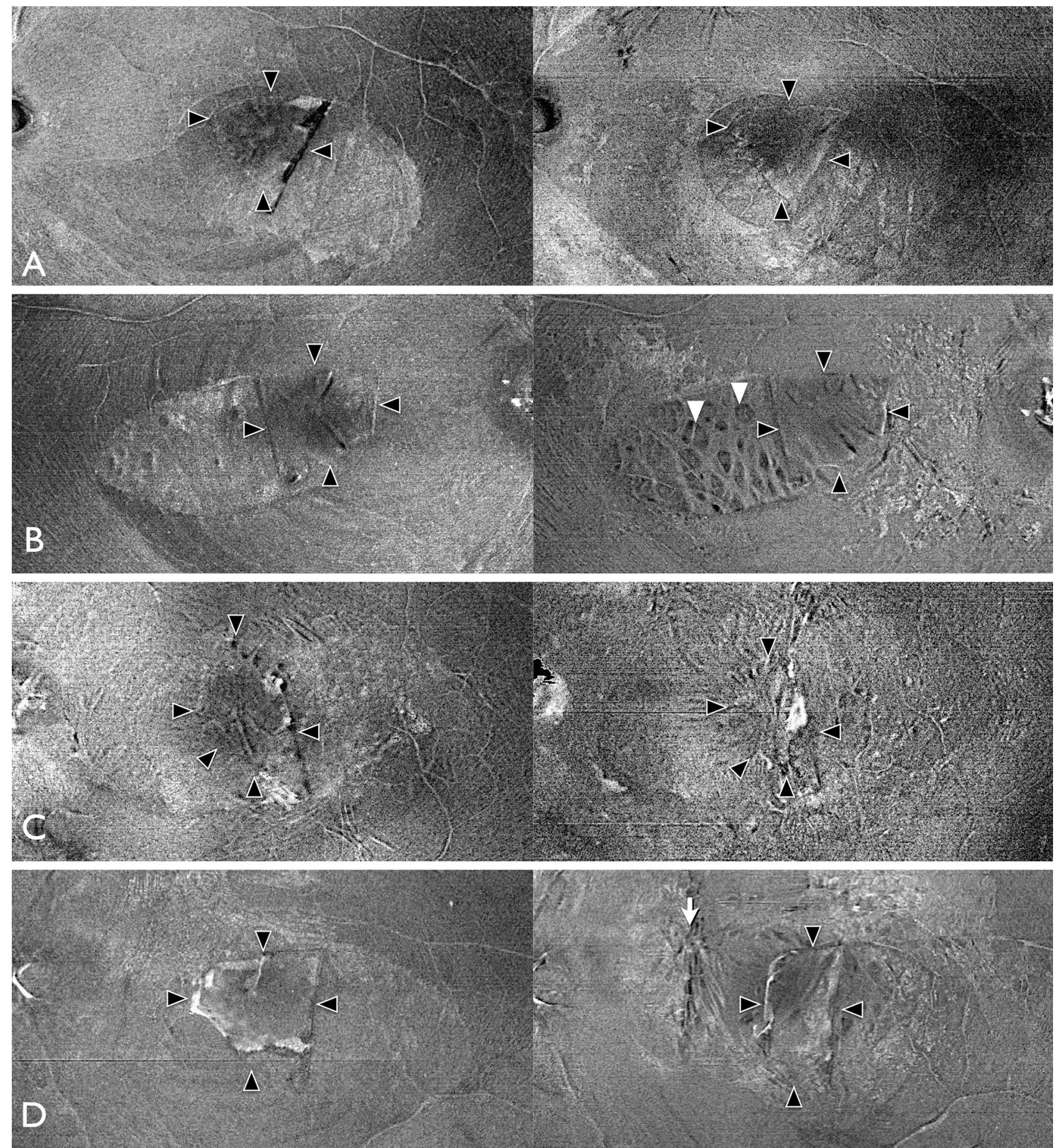

Figure 3. En face images of the internal limiting membrane (ILM) flaps. The left panel shows the findings at 1 month postoperatively, and the right panel shows the findings at 6 months, postoperatively. Cases with little change in the ILM flap area between 1 month and 6 months postoperatively $(\mathbf{A}, \mathbf{B})$ and cases with more than $5 \%$ contraction of the ILM flap at 6 months postoperatively (C,D). (A) A 72-year-old patient with idiopathic MH. The ILM flap over the macular hole $(\mathrm{MH})$ (surrounded by arrowheads) remains exactly the same shape 6 months after surgery. The ILM flap area was $2.192 \mathrm{~mm}^{2}$ at 1 month postoperatively and $2.134 \mathrm{~mm}^{2} 6$ months postoperatively. (B) A 73-year-old patient with idiopathic M. Although the ILM flap (arrowheads) retained the same shape at 6 months postoperatively, the ILM flap showed increased wrinkling. Inner retinal dimpling (white arrowheads) was observed in the area where the ILM was peeled to create the flap. The ILM flap area was $3232 \mathrm{~mm}^{2}$ at 1 month postoperatively and $3262 \mathrm{~mm}^{2} 6$ months postoperatively. (C) A 72-year-old patient with idiopathic MH. The ILM flap (arrowheads) contracted centripetally at 6 months postoperatively, and wrinkles were observed on the retinal surface as the ILM flap contracted. The ILM flap area was $3.543 \mathrm{~mm}^{2}$ at 1 month postoperatively and $3.318 \mathrm{~mm}^{2} 6$ months postoperatively. (D) A 45-year-old patient with myopic MH. The ILM flap (arrowheads) contracted and curled at the nasal side of the flap at 6 months postoperatively; ERM formation was also observed at the edge of the ILM peeled area (arrow). The ILM flap area was $3.601 \mathrm{~mm}^{2}$ at 1 month postoperatively and $2.776 \mathrm{~mm}^{2} 6$ months postoperatively. 


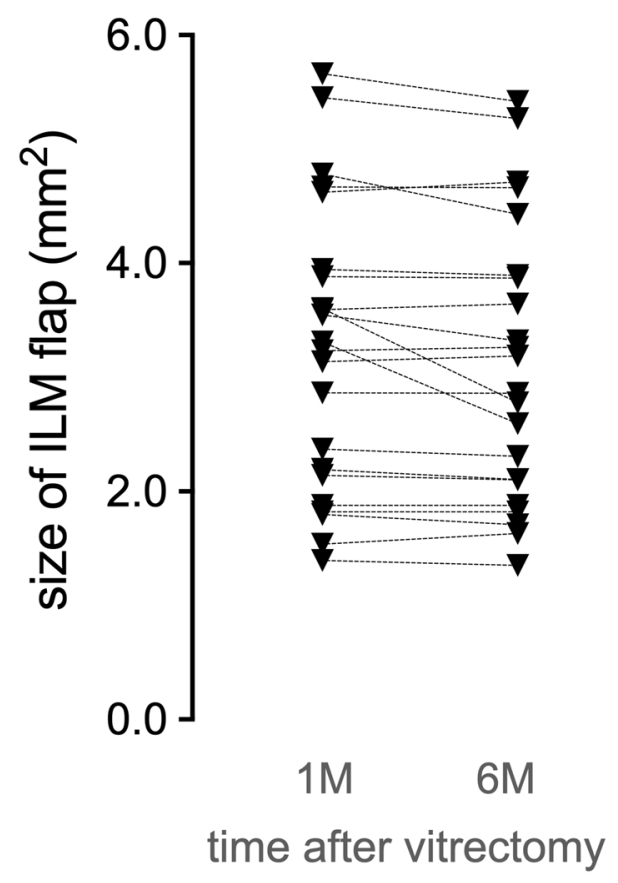

Figure 4. Area of the internal limiting membrane (ILM) flap 1 month and 6 months after surgery. The area of the ILM flap was significantly reduced from $3.25 \pm 1.27 \mathrm{~mm}^{2}$ from 1 month to $3.13 \pm 1.23 \mathrm{~mm}^{2}$ to 6 months $(P=0.024$, Wilcoxon signed rank test). Five eyes ( $23 \%)$ showed $\geq 5 \%$ ILM flap contraction, of which two eyes had $\geq 20 \%$ contraction of ILM flaps.

In this study, we presented pre- and postoperative visual acuity as an index of visual function. Similarly, metamorphopsia is one of the most important complaints after MH surgery and is thought to be related to postoperative macular deformation caused by ILM peeling ${ }^{30-32}$. However, it is not fully understood how the MH coverage by the ILM flap affects postoperative metamorphopsia. Although quantitative evaluation of metamorphopsia was not performed in this study, the experience of one case in which metamorphopsia was worsened by ILM flap contraction suggests that ILM flaps may be involved in postoperative metamorphopsia. The mechanism of the change in metamorphopsia due to the difference in the MH repair process after vitrectomy with the ILM flap and the progression of metamorphopsia due to the contraction of the ILM flap need to be further investigated.

The limitations of this study are the small number of cases included and the relatively short observation period of 6 months. It has been considered that morphological changes of the ILM flap could occur and should be examined over a long period of time.

In conclusion, vitrectomy using the inverted ILM flap technique yielded good anatomical and functional results. However, there are cases in which ILM flap contraction is observed during the postoperative period. Especially in younger individuals and cases with large flaps, the degree of contraction of the ILM flap tends to be large, and reoperation may be required. The ILM flap technique is a relatively new technique, and it is important to find a safe protocol that can be reliably performed, and can facilitate the maintenance of good visual function for a long period of time.

\section{Patients and methods}

Patients. This study included patients who underwent vitreous surgery for $\mathrm{MH}$ at the Hayashi Eye Hospital between August 2018 and February 2020, in which the MH was treated by vitrectomy with coverage of a single ILM flap and had a follow-up period of at least 6 months after vitrectomy. We conducted this prospective observational case series study in accordance with the Declaration of Helsinki and received approval from the Ethics Committee of the Hayashi Eye Hospital. All patients who participated in the study gave written informed consent after receiving an explanation of the nature of the study before inclusion in the study. The ILM flap technique was indicated when the hole diameter was greater than $400 \mu \mathrm{m}$, in the case of idiopathic MHs and myopic MHs, with an axial length of $\geq 26 \mathrm{~mm}$. Eyes with diabetic retinopathy, retinal vein occlusion, a history of pars plana vitrectomy, or uveitis, were excluded.

Surgical procedure. All patients underwent surgery by a single surgeon (A.H.) following induction of local sub-Tenon's anesthesia, as previously reported ${ }^{12,33}$. Phacoemulsification and aspiration (PEA) and 3-piece hydrophobic acrylic intraocular lens (IOL) implantation were performed in 15 patients with cataracts. Three patients had a clear lens that did not require cataract surgery. The remaining five patients had already undergone cataract surgery. Subsequently, we performed a 25-gauge pars plana vitrectomy using a wide-angle viewing system (Fig. 6 and Supplemental Video 3). After core vitrectomy, a posterior vitreous detachment was created in cases where posterior vitreous detachment had not occurred. After staining the macula with Brilliant Blue G 

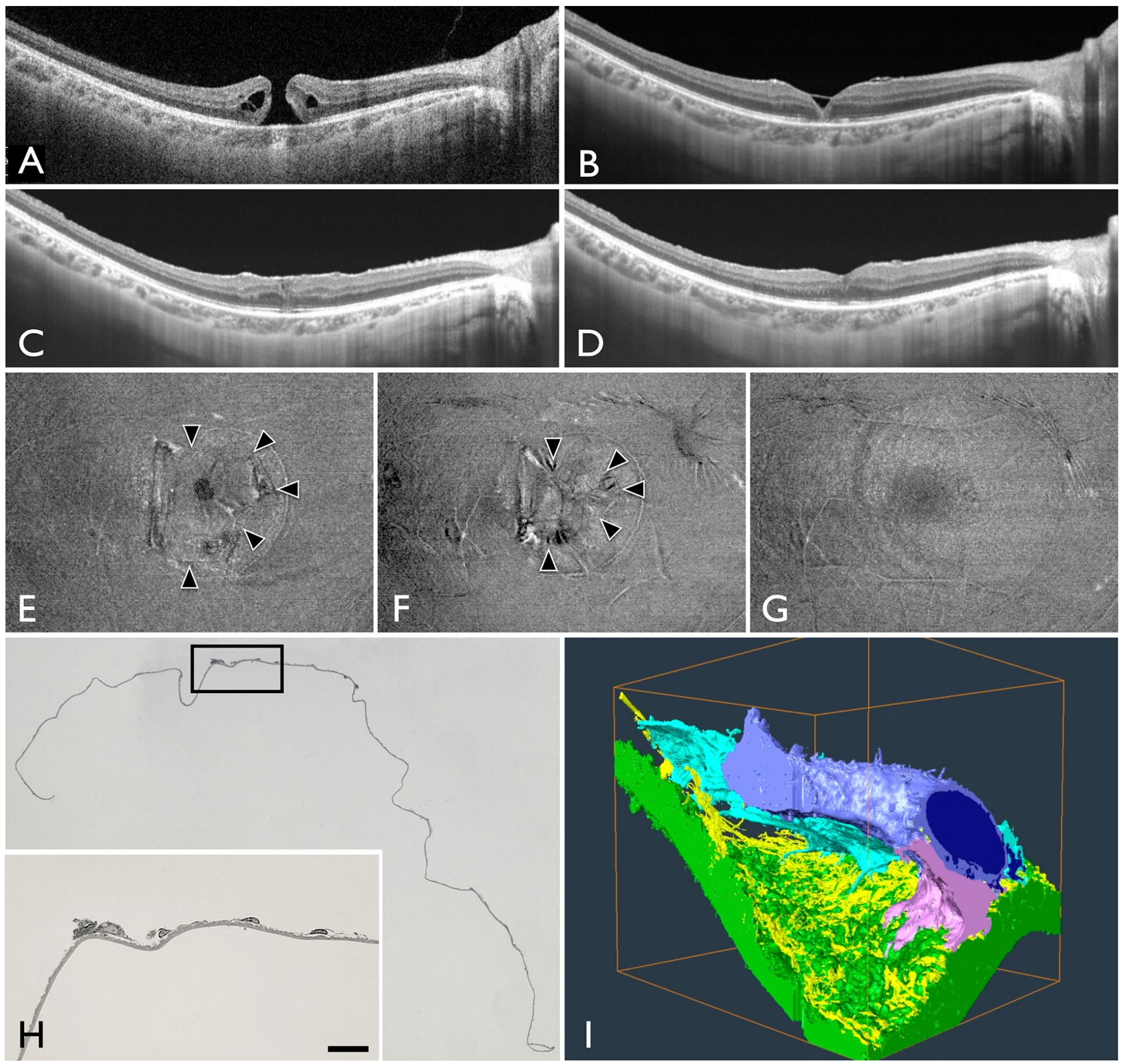

Figure 5. Clinical findings in a 37-year-old patient with myopic macular hole (MH) requiring reoperation. (A) Preoperative OCT image. The MH diameter was $290 \mu \mathrm{m}$ and the axial length was $27.11 \mathrm{~mm}$. (B) OCT image obtained 1 month after the surgery. The ILM flap covered the $\mathrm{MH}$, and the $\mathrm{MH}$ was closed. (C) OCT image obtained 6 months after surgery. Although the patient's vision improved after the vitrectomy, the foveal depression gradually disappeared. (D) OCT image at 13 months after the first surgery (1 month after the second surgery). Twelve months after the first surgery, the patient underwent another vitrectomy to remove the ILM flap. One month after the surgery, the central foveal depression recovered, and BCVA improved. (E) OCT En Face image at 1 month postoperatively; an ILM flap with a clear border was observed covering the macula. The area of the ILM flap was $3.31 \mathrm{~mm}^{2}$. (F) OCT En Face image at 6 months postoperatively. The ILM flap had wrinkles and afferent contraction, and the area of the ILM flap was reduced to $2.59 \mathrm{~mm}^{2}$. (G) OCT En Face image at 13 months after the first surgery ( 1 month after the second surgery). The ILM flap was completely removed. (H) Light microscopy findings of the removed ILM flap. The ILM flap could be peeled off as a single sheet, and cell adhesion was observed on the surface of the ILM. The cells were found on the surface of the ILM that was originally on the retinal side, that is, the area that was newly inverted and faced the vitreous side. Bar, $10 \mu \mathrm{m}$. (I) Three-dimensional reconstructed image of the peeled ILM flap using focused ion beam-equipped scanning electron microscopy, as described previously ${ }^{36}$. Multiple cells (purple, pink, and sky blue) adhered to the ILM (green) surface, and cell-cell adhesion was also observed. The fibrous component (yellow) is also seen on the surface of the ILM. 


\begin{tabular}{|l|l|l|l|l|l|l|}
\hline Variables & $\begin{array}{l}\text { Partial regression } \\
\text { coefficient }\end{array}$ & Standard error & t-value & P-value & 95\% CI & $\begin{array}{l}\text { Standardized partial } \\
\text { regression coefficient }\end{array}$ \\
\hline Intercept & 73.94 & 6.39 & 11.58 & $<0.001$ & 60.57 to 87.31 & \\
\hline Age (years) & 0.46 & 0.10 & 4.49 & $<0.001$ & 0.24 to 0.69 & 0.74 \\
\hline $\begin{array}{l}\text { Size of ILM flap at } \\
1 \text { month }\left(\mathrm{mm}^{2}\right)\end{array}$ & -1.97 & 0.89 & -2.20 & 0.040 & -3.84 to -0.10 & -0.36 \\
\hline
\end{tabular}

Table 2. Clinical factors related to ILM flap contraction. Multiple linear regression analysis with the contraction rate of the ILM flap as the objective variables and the selected preoperative clinical data as explanatory variables. ILM internal limiting membrane.

solution $^{34}$, the ILM was peeled at the size of one disc diameter around the MH (Fig. 6A). Next, an ILM flap was created on the temporal side of the macula to sufficiently cover the hole (Fig. 6B), and the flap was stretched and covered over the hole using dispersive ophthalmic viscosurgical devices (Fig. 6C). Peripheral vitrectomy was then performed while monitoring for peripheral retinal tears. Finally, fluid-air exchange and air tamponade were performed (Fig. 6D). The patient was instructed to remain in the prone position for 1-3 days postoperatively.

Examination. Best-corrected visual acuity (BCVA) was measured using the Landolt chart at various time points including, before surgery, immediately after the disappearance of air in the eye, and at three post-operative points $(1,3$, and 6 months). BCVA measurements were converted to the logarithm of the minimum angle of resolution (logMAR) for analysis. All patients underwent retinal imaging using a swept-source OCT (Plex Elite 9000; Carl Zeiss Meditec Inc., Dublin, CA, USA). An image with a signal strength of 7 or higher (up to 10) was defined as a suitable. Horizontal and vertical HD scans (HD Spotlight 1 with a measurement width of $16 \mathrm{~mm}$, $0^{\circ}$, and $90^{\circ}$ ) were used to measure the $\mathrm{MH}$ diameter and to analyze the continuity of the ELM and ellipsoid zone. Cube scans $(12 \mathrm{~mm} \times 12 \mathrm{~mm}, 800 \times 800$ pixels $)$ were used to measure the size of the ILM flap. En face structural slabs of the vitreoretinal interface were created from the cube scans by automated segmentation $(133 \mu \mathrm{m}$ above the ILM layer as the inner boundary and $33 \mu \mathrm{m}$ below the ILM layer as the outer boundary) allowing visualization of the ILM flap boundary. The en face image obtained to measure the size of the ILM flap was saved as a TIFF file and imported with ImageJ software (https://imagej.nih.gov/ij/; provided in the public domain by the National Institutes of Health, Bethesda, MD) ${ }^{35}$. The boundaries of the ILM flaps were manually traced and segmented using a graphics tablet (Wacom Cintiq 16; Wacom Co., Ltd., Kazo, Saitama, Japan), and the flap area was measured using the measurement features built into ImageJ.

Before the measurement of the ILM flap area, a reconstructed image of the retinal surface was created using $3 \mathrm{D}$ reconstruction software to verify whether the contour of the ILM flap, obtained from the en face image of OCT, was accurate. The captured data from the cube scan were exported as a movie file, processed, and saved as a stack image using ImageJ. The data were further transferred to the $3 \mathrm{D}$ visualization/analysis software (FEI Amira v6.0.1 for Mac OS X; https://www.thermofisher.com/jp/en/home/electron-microscopy/products/softw are-em-3d-vis/amira-software.html; Thermo Fisher Scientific, Waltham, MA), and a 3D image of the macular retina was created. The obtained images showed the unevenness of the retinal surface from various directions, and the contour of the ILM was confirmed to be consistent with the contour of the ILM flap obtained from the en face image (Supplemental Video 1).

The following four issues were examined: postoperative $\mathrm{MH}$ status (presence or absence of $\mathrm{MH}$ closure and the continuity of external limiting membrane and ellipsoid zone), comparison of BCVA before and 6 months after surgery, comparison of the ILM flap area at 1 month and 6 months postoperatively, and analysis of preoperative clinical data related to changes in the ILM flap size.

Specimen preparation and focused ion beam equipped scanning electron microscope (FIB/ SEM) tomography examination. In one case that required reoperation, the ILM flap removed during surgery was immediately immersion-fixed in $2.5 \%$ glutaraldehyde solution in $0.1 \mathrm{M}$ phosphate-buffered saline (PBS) for at least $2 \mathrm{~h}$ and then stored for further preparation. The specimen was prepared as previously described $^{36,37}$. Briefly, after washing five times with PBS, the specimen was postfixed for $30 \mathrm{~min}$ in a solution containing $2 \%$ osmium tetroxide and $1.5 \%$ potassium ferrocyanide in PBS at $4{ }^{\circ} \mathrm{C}$. The specimen was then washed with distilled water and immersed in $1 \%$ thiocarbohydrazide solution for $30 \mathrm{~min}$. After washing with distilled water, the specimen was further immersed in $2 \%$ osmium tetroxide in distilled water and then washed with distilled water. Subsequently, the specimen was en bloc stained in a solution of $4 \%$ uranyl acetate solution overnight and further stained by Walton's lead aspartate solution for $1 \mathrm{~h}$ for contrast enhancement. After the staining, the specimen was dehydrated in an ethanol series, followed by infiltration of epoxy resin (Epon 812; TAAB Laboratories Equipment Ltd., Berkshire, UK) mixture, and polymerized for $72 \mathrm{~h}$ at $60{ }^{\circ} \mathrm{C}$. The surface of the embedded specimen was exposed using a diamond knife on an Ultracut E microtome (Leica, Wetzlar, Germany). The resin block was then placed on a standard scanning electron microscope (SEM) specimen holder with adhesives for SEM imaging.

The freshly exposed surface of the specimen was examined by backscattered electron imaging using FIB/ SEM (Quanta Three-dimensional FEG, FEI, Eindhoven, The Netherlands). Serial images of the block face were acquired by repeated cycles of sample surface milling and imaging using Slice and View G2 software (FEI). The milling pitch was set to $50 \mathrm{~nm} / \mathrm{step}$ and 800 cycles. The resultant image stack was processed using the ImageJ 

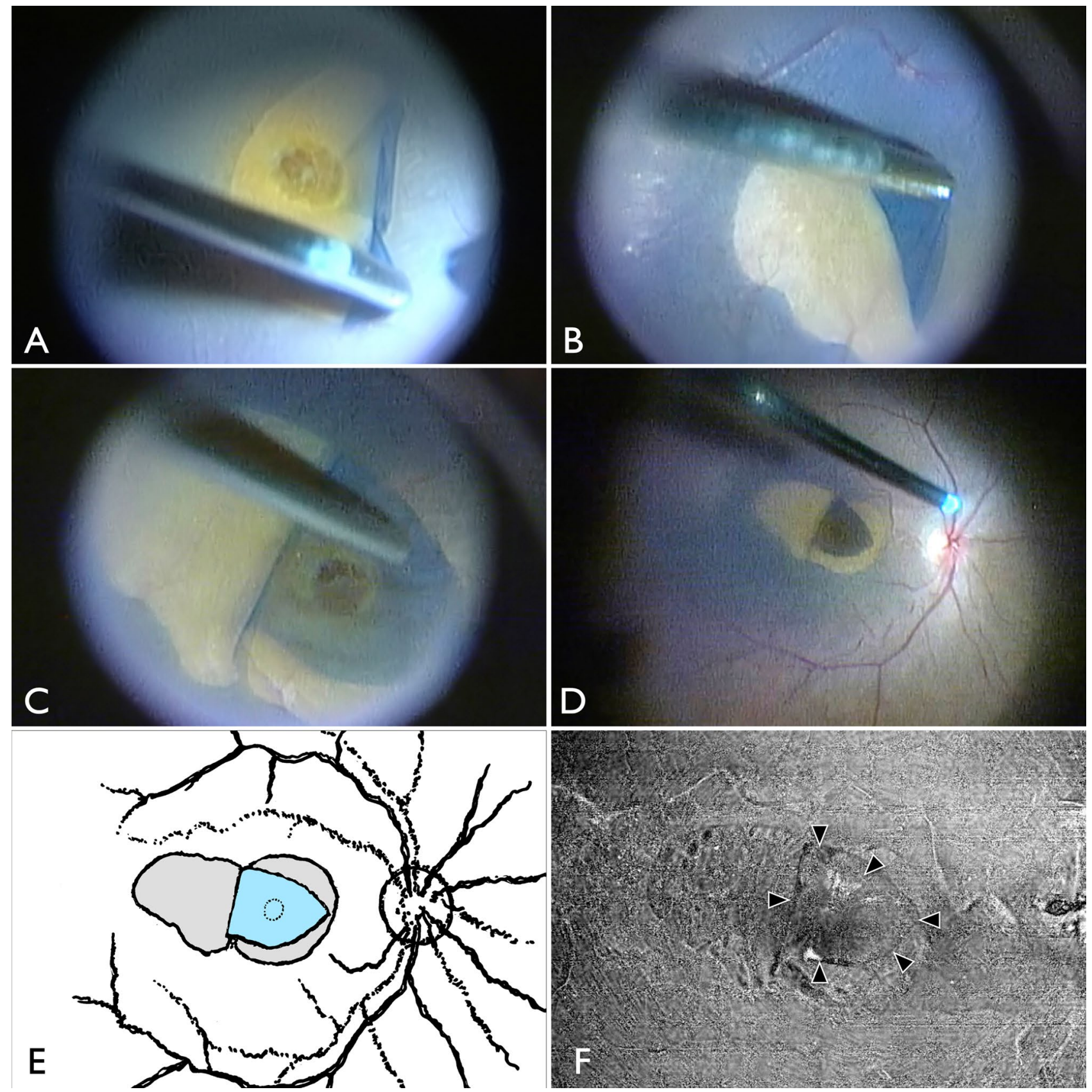

Figure 6. Intraoperative findings demonstrating surgical technique. (A) After core vitrectomy with posterior vitreous detachment, internal limiting membrane (ILM) peeling around the macular hole (MH) was performed with a radius of a one disc diameter. (B) An ILM flap was created at a temporal site approximately 2DD away from the ILM peeling edge, with the temporal ILM peeling edge as a hinge. (C) The ILM flap covered over the $\mathrm{MH}$, and dispersive ophthalmic viscosurgical devices were applied to fix the ILM flap. (D) Fluid-air exchange was performed to fill the vitreous cavity with air. (E) Schematic diagram of surgical findings: ILM flap is shown in sky blue, ILM peeling area and ILM flap fabrication site are shown in gray. (F) OCT en face image at 6 months postoperatively. The ILM flap that was covered at the time of surgery was clearly observed.

(National Institutes of Health, Bethesda, MD) and FEI Amira v6.0.1 software (Thermo Fisher Scientific, Waltham, MA). To observe the three-dimensional structures of the specimen, the ILM and the membrane components of the cells were semi-automatically segmented. Subsequently, the outlined ILM and cells were then visualized and displayed.

Statistical analysis. In comparing the clinical characteristics of patients with idiopathic MH and myopic $\mathrm{MH}$, we used an unpaired $t$ test for patient age, MH size, preoperative BCVA, and axial length, and a Fisher's exact probability test for sex. Comparison of pre-and postoperative BCVA was performed using the paired $t$ test, 
and the ILM flap area was compared using en face images at the vitreoretinal interface and tested using the Wilcoxon signed-rank test. The Spearman's rank correlation coefficient was calculated for the correlation between postoperative BCVA after 6 months and preoperative factors including patients age, sex, $\mathrm{MH}$ size, preoperative BCVA and axial length, and the size of the ILM flap at 1 month. The factors related to ILM flap contraction were analyzed by multiple linear regression analysis with the objective variables being the contraction rate of the ILM flap and the preoperative clinical data selected by the stepwise method as explanatory variables. All statistical analyses were performed using JMP Pro 15 statistical software (SAS Institute, Inc., Cary, NC, USA) or Prism 7 for Mac OS X (version 7.0b, GraphPad Software, Inc., San Diego, CA, USA), and statistical significance was set at $P<0.05$.

\section{Data availability}

The datasets used and/or analyzed during the current study are available from the corresponding author on reasonable request.

Received: 12 June 2021; Accepted: 28 September 2021

Published online: 08 October 2021

\section{References}

1. Kelly, N. E. \& Wendel, R. T. Vitreous surgery for idiopathic macular holes. Results of a pilot study. Arch. Ophthalmol. 109, 654-659. https://doi.org/10.1001/archopht.1991.01080050068031 (1991).

2. Eckardt, C., Eckardt, U., Groos, S., Luciano, L. \& Reale, E. Removal of the internal limiting membrane in macular holes. Clinical and morphological findings. Ophthalmologe 94, 545-551. https://doi.org/10.1007/s003470050156 (1997).

3. Kumagai, K. et al. Vitreous surgery with and without internal limiting membrane peeling for macular hole repair. Retina 24, 721-727. https://doi.org/10.1097/00006982-200410000-00006 (2004).

4. Michalewska, Z., Michalewski, J., Adelman, R. A. \& Nawrocki, J. Inverted internal limiting membrane flap technique for large macular holes. Ophthalmology 117, 2018-2025. https://doi.org/10.1016/j.ophtha.2010.02.011 (2010).

5. Boninska, K., Nawrocki, J. \& Michalewska, Z. Mechanism of "flap closure" after the inverted internal limiting membrane flap technique. Retina 38, 2184-2189. https://doi.org/10.1097/IAE.0000000000001861 (2018).

6. Kuriyama, S. et al. Efficacy of inverted internal limiting membrane flap technique for the treatment of macular hole in high myopia. Am. J. Ophthalmol. 156, 125-131 e121. https://doi.org/10.1016/j.ajo.2013.02.014 (2013).

7. Baba, R. et al. Efficacy of the inverted internal limiting membrane flap technique with vitrectomy for retinal detachment associated with myopic macular holes. Retina 37, 466-471. https://doi.org/10.1097/IAE.0000000000001211 (2017).

8. Hu, X. T., Pan, Q. T., Zheng, J. W. \& Zhang, Z. D. Foveal microstructure and visual outcomes of myopic macular hole surgery with or without the inverted internal limiting membrane flap technique. Br. J. Ophthalmol. 103, 1495-1502. https://doi.org/10.1136/ bjophthalmol-2018-313311 (2019).

9. Michalewska, Z., Michalewski, J., Dulczewska-Cichecka, K. \& Nawrocki, J. Inverted internal limiting membrane flap technique for surgical repair of myopic macular holes. Retina 34, 664-669. https://doi.org/10.1097/IAE.0000000000000042 (2014).

10. Michalewska, Z., Michalewski, J., Dulczewska-Cichecka, K., Adelman, R. A. \& Nawrocki, J. Temporal inverted internal limiting membrane flap technique versus classic inverted internal limiting membrane flap technique: A comparative study. Retina 35, 1844-1850. https://doi.org/10.1097/IAE.0000000000000555 (2015).

11. Shin, M. K., Park, K. H., Park, S. W., Byon, I. S. \& Lee, J. E. Perfluoro-n-octane-assisted single-layered inverted internal limiting membrane flap technique for macular hole surgery. Retina 34, 1905-1910. https://doi.org/10.1097/IAE.0000000000000339 (2014).

12. Hirata, A., Takano, A., Kawaji, T. \& Nakamura, K. I. Macular pucker formation after macular hole surgery with inverted internal limiting membrane flap technique and silicone oil tamponade. Am. J. Ophthalmol. Case Rep. 19, 100847. https://doi.org/10.1016/j. ajoc.2020.100847 (2020).

13. Shiode, Y. et al. The role of inverted internal limiting membrane flap in macular hole closure. Investig. Ophthalmol. Vis. Sci. 58, 4847-4855. https://doi.org/10.1167/iovs.17-21756 (2017).

14. Baumann, C. et al. Effect of inverted internal limiting membrane flap on closure rate, postoperative visual acuity, and restoration of outer retinal layers in primary idiopathic macular hole surgery. Retina https://doi.org/10.1097/IAE.00000000000002707 (2019).

15. Pak, K. Y., Park, J. Y., Park, S. W., Byon, I. S. \& Lee, J. E. Efficacy of the perfluoro- $N$-octane-assisted single-layered inverted internal limiting membrane flap technique for large macular holes. Ophthalmologica 238, 133-138. https://doi.org/10.1159/000477823 (2017).

16. Ramtohul, P., Parrat, E., Denis, D. \& Lorenzi, U. Inverted internal limiting membrane flap technique versus complete internal limiting membrane peeling in large macular hole surgery: A comparative study. BMC Ophthalmol. 20, 11. https://doi.org/10.1186/ s12886-019-1294-8 (2020).

17. Iwasaki, M., Kinoshita, T., Miyamoto, H. \& Imaizumi, H. Influence of inverted internal limiting membrane flap technique on the outer retinal layer structures after a large macular hole surgery. Retina 39, 1470-1477. https://doi.org/10.1097/IAE.0000000000 002209 (2019).

18. Rossi, T. et al. Macular hole closure patterns associated with different internal limiting membrane flap techniques. Graefes Arch. Clin. Exp. Ophthalmol. 255, 1073-1078. https://doi.org/10.1007/s00417-017-3598-9 (2017).

19. Park, J. H., Lee, S. M., Park, S. W., Lee, J. E. \& Byon, I. S. Comparative analysis of large macular hole surgery using an internal limiting membrane insertion versus inverted flap technique. Br. J. Ophthalmol. 103, 245-250. https://doi.org/10.1136/bjophthalm ol-2017-311770 (2019).

20. Faria, M. Y. et al. Inverted internal limiting membrane flap techniques and outer retinal layer structures. Retina https://doi.org/ 10.1097/IAE.0000000000002607 (2019).

21. Gekka, T. et al. Pedicle internal limiting membrane transposition flap technique for refractory macular hole. Ophthalmic Surg. Lasers Imaging Retina 46, 1045-1046. https://doi.org/10.3928/23258160-20151027-10 (2015).

22. Chen, S. N. Large semicircular inverted internal limiting membrane flap in the treatment of macular hole in high myopia. Graefes Arch. Clin. Exp. Ophthalmol. 255, 2337-2345. https://doi.org/10.1007/s00417-017-3808-5 (2017).

23. Aurora, A., Seth, A. \& Sanduja, N. Cabbage leaf inverted flap ILM peeling for macular hole: A novel technique. Ophthalmic Surg. Lasers Imaging Retina 48, 830-832. https://doi.org/10.3928/23258160-20170928-08 (2017).

24. Tabandeh, H., Morozov, A., Rezaei, K. A. \& Boyer, D. S. Superior wide-base internal limiting membrane flap transposition for macular holes: Flap status and outcomes. Ophthalmol. Retina 5, 317-323. https://doi.org/10.1016/j.oret.2020.12.003 (2021).

25. Takai, Y., Tanito, M., Sugihara, K. \& Ohira, A. The role of single-layered flap in temporal inverted internal limiting membrane flap technique for macular holes: Pros and cons. J. Ophthalmol. 2019, 5737083. https://doi.org/10.1155/2019/5737083 (2019).

26. De Novelli, F. J. et al. Recurrence rate and need for reoperation after surgery with or without internal limiting membrane removal for the treatment of the epiretinal membrane. Int. J. Retina Vitreous 3, 48. https://doi.org/10.1186/s40942-017-0101-z (2017). 
27. Sandali, O. et al. Epiretinal membrane recurrence: Incidence, characteristics, evolution, and preventive and risk factors. Retina 33, 2032-2038. https://doi.org/10.1097/IAE.0b013e31828d2fd6 (2013).

28. Kwok, A., Lai, T. Y. \& Yuen, K. S. Epiretinal membrane surgery with or without internal limiting membrane peeling. Clin. Exp. Ophthalmol. 33, 379-385. https://doi.org/10.1111/j.1442-9071.2005.01015.x (2005).

29. Gandorfer, A. et al. Residual cellular proliferation on the internal limiting membrane in macular pucker surgery. Retina 32, 477-485. https://doi.org/10.1097/IAE.0b013e3182246e2a (2012).

30. Bae, K. et al. Extent of internal limiting membrane peeling and its impact on macular hole surgery outcomes: A randomized trial. Am. J. Ophthalmol. 169, 179-188. https://doi.org/10.1016/j.ajo.2016.06.041 (2016).

31. Akahori, T. et al. Macular displacement after vitrectomy in eyes with idiopathic macular hole determined by optical coherence tomography angiography. Am. J. Ophthalmol. 189, 111-121. https://doi.org/10.1016/j.ajo.2018.02.021 (2018).

32. Park, S. H. et al. Square grid deformation analysis of the macula and postoperative metamorphopsia after macular hole surgery. Retina 41, 931-939. https://doi.org/10.1097/IAE.0000000000002955 (2021).

33. Hirata, A., Hayashi, K., Murata, K. \& Nakamura, K. I. Removal of choroidal neovascular membrane in a case of macular hole after anti-VEGF therapy for age-related macular degeneration. Am. J. Ophthalmol. Case Rep. 9, 14-17. https://doi.org/10.1016/j.ajoc. 2017.12.003 (2018).

34. Enaida, H. et al. Preclinical investigation of internal limiting membrane staining and peeling using intravitreal brilliant blue G. Retina 26, 623-630. https://doi.org/10.1097/01.iae.0000236470.71443.7c (2006).

35. Schneider, C. A., Rasband, W. S. \& Eliceiri, K. W. NIH Image to ImageJ: 25 years of image analysis. Nat. Methods 9, 671-675. https://doi.org/10.1038/nmeth.2089 (2012).

36. Hirata, A., Murata, K., Hayashi, K. \& Nakamura, K. I. Three-dimensional analysis of peeled internal limiting membrane using focused ion beam/scanning electron microscopy. Transl. Vis. Sci. Technol. 7, 15. https://doi.org/10.1167/tvst.7.1.15 (2018).

37. Murata, K., Hirata, A., Ohta, K., Enaida, H. \& Nakamura, K. I. Morphometric analysis in mouse scleral fibroblasts using focused ion beam/scanning electron microscopy. Sci. Rep. 9, 6329. https://doi.org/10.1038/s41598-019-42758-x (2019).

\section{Acknowledgements}

The authors thank Editage (http://www.editage.jp) for English language editing.

\section{Author contributions}

A.H. designed the study and wrote the main manuscript text. K.M. contributed to data collection and interpretation. K.H. contributed to analysis and interpretation of data, and assisted in the preparation of the manuscript. All authors approved the final version of the manuscript.

\section{Funding}

This work was supported by JSPS KAKENHI, Grant Number JP 19K10003.

\section{Competing interests}

The authors declare no competing interests.

\section{Additional information}

Supplementary Information The online version contains supplementary material available at https://doi.org/ 10.1038/s41598-021-99509-0.

Correspondence and requests for materials should be addressed to A.H.

Reprints and permissions information is available at www.nature.com/reprints.

Publisher's note Springer Nature remains neutral with regard to jurisdictional claims in published maps and institutional affiliations.

(c) (i) Open Access This article is licensed under a Creative Commons Attribution 4.0 International

License, which permits use, sharing, adaptation, distribution and reproduction in any medium or format, as long as you give appropriate credit to the original author(s) and the source, provide a link to the Creative Commons licence, and indicate if changes were made. The images or other third party material in this article are included in the article's Creative Commons licence, unless indicated otherwise in a credit line to the material. If material is not included in the article's Creative Commons licence and your intended use is not permitted by statutory regulation or exceeds the permitted use, you will need to obtain permission directly from the copyright holder. To view a copy of this licence, visit http://creativecommons.org/licenses/by/4.0/.

(c) The Author(s) 2021 